\title{
Fever of Unknown Origin — infected Fistula-in-Ano as the focus on ${ }^{18}$ F-FDG PET-CT
}

Punit Sharma

Department of Nuclear Medicine and PET-CT, Apollo Gleneagles Hospitals, Kolkata, India

[Received 14 VII 2020; Accepted 22 XII 2020]

\section{Abstract}

Fever of Unknown Origin (FUO) is a vexing clinical problem. Diagnosis of aetiology is essential for definitive management. A wide array of infective, inflammatory, malignant and miscellaneous pathologies can cause FUO. Hybrid imaging with $18 \mathrm{~F}$-Fluorodeoxyglucose $\left({ }^{18} \mathrm{~F}-\mathrm{FDG}\right)$ positron emission tomography-computed tomography (PET-CT) is now an integral part of FUO management because of its ability to demonstrate the cause in a large proportion of cases. The authors present the case of a 42-year-old male, where an infected fistula-in-ano was detected as the cause of FUO on ${ }^{18} \mathrm{~F}$-FDG PET-CT.

KEY words: FUO; FDG; PET-CT; fistula-in-ano

Nucl Med Rev 2021; 24, 1: 31-32

A 42-year-old male presented at the hospital with complaints of multiple episodes of low to moderate grade fever for the past 6 months. The fever subsided either spontaneously or with empirical antibiotic therapy. He was hypertensive but had no other known comorbidity. The episode this time was for 7-day duration, with peak fever of $101^{\circ} \mathrm{F}$. There were no localising symptoms. Clinical examination was unremarkable. Blood parameters were all normal, except for a mild neutrophilic leucocytosis (Total leucocyte count-12.000/mL; 91\% Neutrophils) and raised C-reactive protein (25 mg/L). 18F-Fluorodeoxyglucose ( $\left.{ }^{18} \mathrm{~F}-\mathrm{FDG}\right)$ positron emission tomography-computed tomography (PET-CT) (Fig. 1) was done to localise the cause of fever. Maximum intensity projection (MIP) PET images (A) showed focal increased radiotracer uptake in lower pelvis in the midline (arrow), apart from left renal pelviectasis (broken arrow). Sectional CT and PET-CT (B-G) images showed an ${ }^{18} \mathrm{~F}-\mathrm{FDG}$ avid tract (arrow) extending from the anal sphincter to perianal skin posteriorly in the midline in subcutaneous plane, with some fat stranding around the distal part (SUV max 9.1). Findings suggested an infected fistula-in-ano. Subsequent local examination revealed no definite cutaneous opening of fistula but a focal scar in this region and mild tenderness. On anoscopy, small internal haemorrhoids were seen. Also noted was the internal opening of the fistula. The patient was started on antibiotics and became afebrile. Magnetic resonance imaging (MRI) was performed later which confirmed infra-levator translate-sphincteric fistula and underwent surgery for the same.

Fistula-in-ano is an abnormal communication between the anal canal and the perianal skin. It originates in anal glands, which when blocked and infected form an abscess which then opens into the adjacent skin, forming a fistula. The patients usually present with discharge, pain, tenderness and at times fever. Diagnosis is made with clinical examination and anoscopy, while MRI is the best modality for mapping the fistula tract before surgery, which is the treatment of choice. ${ }^{18} \mathrm{~F}$-FDG PET-CT is now an integral part of FUO management for localising the cause. Because of the non-specific nature of ${ }^{18} \mathrm{~F}-\mathrm{FDG}$, it is taken up by leukocytes as well as malignant cells, thereby allowing demonstration of a wide range of malignant, infective and inflammatory diseases which could present with FUO. In the present case, an infected fistula-in-ano was demonstrated as the cause of fever. 

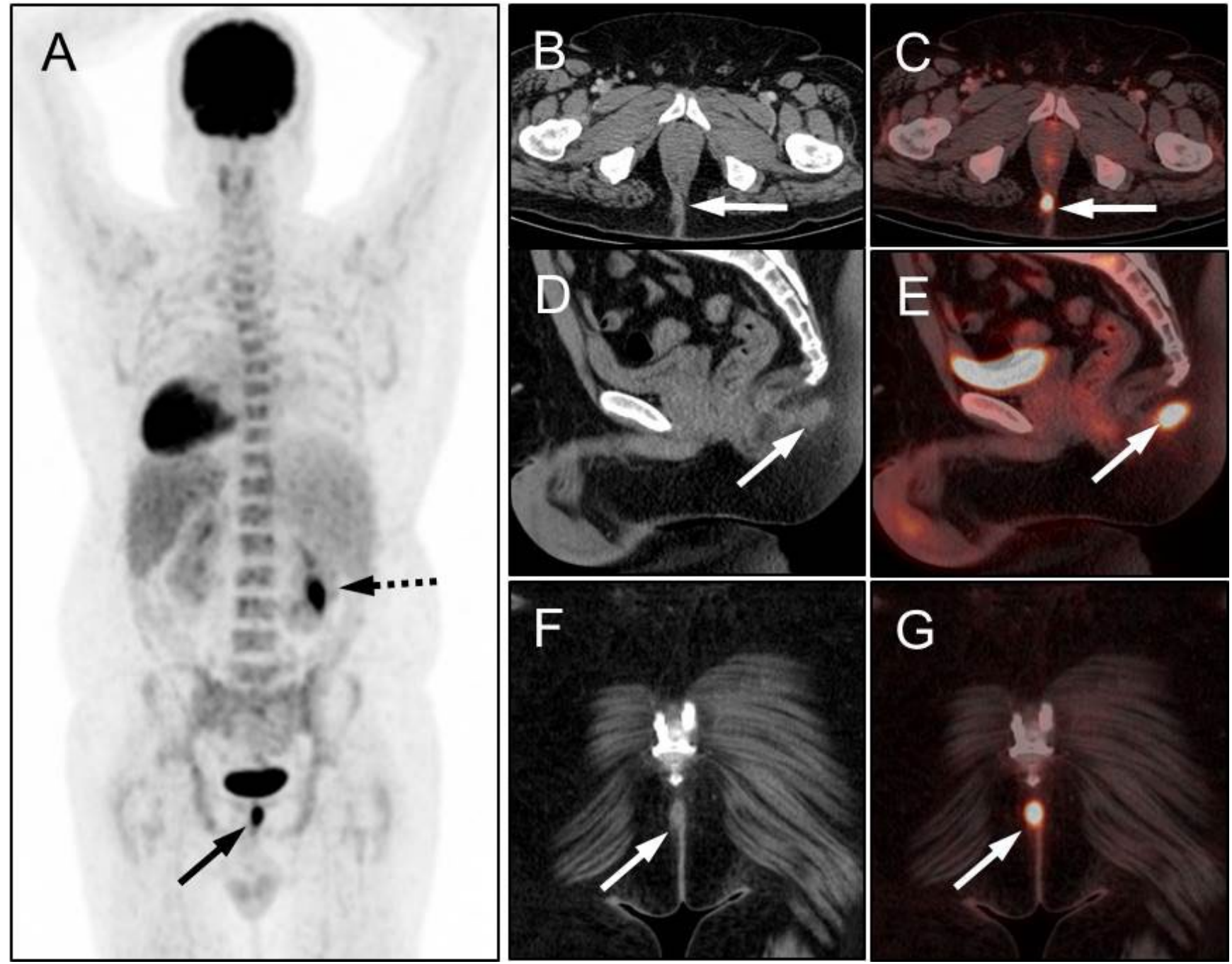

Figure 1. Images from a computer tomograph 